\title{
Generalized stability of classical polynomial functional equation of order $n$
}

\author{
Tippaporn Eungrasamee, Patanee Udomkavanich and Paisan Nakmahachalasint
}

"Correspondence:

Paisan.N@chula.ac.th

Department of Mathematics and

Computer Science, Faculty of

Science, Chulalongkorn University,

Bangkok, 10330, Thailand

\begin{abstract}
We study a general $n$th order polynomial functional equation $\Delta_{y}^{n} f(x)=n ! f(y)$ on linear spaces and prove its generalized stability.
\end{abstract}

MSC: 39B52; 39B82

Keywords: polynomial functional equation; generalized stability

\section{Introduction}

The problem of the stability of functional equations started in 1940 by S. M. Ulam [1] when he proposed the question 'Let $G_{1}$ be a group and let $G_{2}$ be a metric group with the metric d. Given $\varepsilon>0$, does there exist a $\delta>0$ such that iff $: G_{1} \rightarrow G_{2}$ satisfies the inequality $d(f(x y), f(x) f(y))<\delta$ for all $x, y \in G_{1}$, then there exists a homomorphism $H: G_{1} \rightarrow G_{2}$ with $d(f(x), H(x))<\varepsilon$ for all $x \in G_{1}$ ?' In the following year, the answer of this question for the case of a mapping $f$ between Banach spaces $E_{1}$ and $E_{2}$ satisfying $\|f(x+y)-f(x)-f(y)\| \leq \varepsilon$ for all $x, y \in E_{1}$ and for some $\varepsilon>0$ was attested by D. H. Hyers [2]. It was shown that for each $x \in E_{1}$, the additive mapping $A: E_{1} \rightarrow E_{2}$ defined by $A(x)=\lim _{n \rightarrow \infty} 2^{-n} f\left(2^{n} x\right)$ has a property that $\|A(x)-f(x)\| \leq \varepsilon$ for all $x \in E_{1}$. Furthermore, the mapping $A$ is also unique. Since then, this kind of stability was known as the Hyers-Ulam stability and became a fundamental stability theory concept of functional equations. In 1950, T. Aoki [3] published a paper on the stability of the additive mappings in Banach spaces, while in 1978, Th. M. Rassias [4] extended the problem to $\|f(x+y)-f(x)-f(y)\| \leq \varepsilon\left(\|x\|^{p}+\|y\|^{p}\right), x, y \in E_{1}$, for some $\varepsilon \geq 0$ and some $0 \leq p<1$. Subsequently, in 1994, P. Gavruta [5] generalized the problem to $\|f(x+y)-f(x)-f(y)\| \leq \phi(x, y)$ with certain conditions imposed on the function $\phi$. This type of stability is referred to as the generalized stability.

In recent years, a number of researchers $[6,7]$ have investigated stability problem of various types of functional equations which are mostly based on the Cauchy additive functional equation of the form $f(x+y)=f(x)+f(y)$, the classical quadratic functional equation $f(x+y)+f(x-y)=2 f(x)+2 f(y)[8]$, and the functional equations of higher degree $[9,10]$. The stability problem of functional equations can be determined on various domains of functions. There were related efforts on functions being defined on groupoid. A typical work was carried out by A. Gilányi [11] in 1999, who proved the Hyers-Ulam stability of monomial functional equation on a power-associative, power-symmetric groupoid. Such efforts investigate a viable further investigation on generalizing the stability problem.

(c) 2012 Eungrasamee et al; licensee Springer. This is an Open Access article distributed under the terms of the Creative Commons Attribution License (http://creativecommons.org/licenses/by/2.0), which permits unrestricted use, distribution, and reproduction in any medium, provided the original work is properly cited. 
In this paper, we will prove the general solution of functional equations of the form

$$
\Delta_{y}^{n} f(x)=n ! f(y)
$$

which is the diagonalization of a symmetric $n$-additive function. We will refer to (1) in this paper as a classical polynomial functional equation of order $n$. (1) was called a monomial functional equation in [11]. Proof of generalized stability of functional equation based on (1) is elucidated as follows. If $n$ is a positive integer, $X$ is a linear space, $Y$ is a Banach space, and a function $f: X \rightarrow Y$ satisfies

$$
\left\|\Delta_{y}^{n} f(x)-n ! f(y)\right\| \leq \phi(x, y)
$$

where $\phi: X^{2} \rightarrow[0, \infty)$ is a function which satisfies certain conditions, then there exists a unique function $A^{n}: X \rightarrow Y$ satisfying (1) and

$$
\left\|f(x)-A^{n}(x)\right\| \leq \sum_{i=0}^{\infty} \frac{\tau\left(2^{i} x\right)}{2^{i n}},
$$

where $\tau: X \rightarrow[0, \infty)$ is a function related to the function $\phi$.

\section{Basic theorems and lemmas}

In this section, we provide some basic theorems and lemmas concerning the difference operators as well as multi-additive functions. For further details and proofs, please refer to the book by S. Czerwik [12]. Throughout the section, we shall let $X$ and $Y$ be two linear spaces and let $f: X \rightarrow Y$ be an arbitrary function.

Definition 1 Define the difference operator $\Delta_{h}$ with the span $h \in X$ by

$$
\Delta_{h} f(x)=f(x+h)-f(x) \quad \text { for all } x \in X .
$$

For each positive integer $n$, we define the iterates $\Delta_{h}^{n}$ by the recurrence

$$
\Delta_{h}^{n+1} f=\Delta_{h}\left(\Delta_{h}^{n} f\right)
$$

We may also write the iterated operators $\Delta_{h_{1}} \cdots \Delta_{h_{n}}$ shortly as $\Delta_{h_{1} \cdots h_{n}}$.

Some properties of the difference operator are shown in the following lemmas.

Lemma 1 The difference operators commute; that is, for all $h_{1}, h_{2} \in X$,

$$
\Delta_{h_{1}} \Delta_{h_{2}} f=\Delta_{h_{2}} \Delta_{h_{1}} f
$$

Lemma 2 Let $n$ be a positive integer. Then, for all $x, h \in X$,

$$
\Delta_{h}^{n} f(x)=\sum_{k=0}^{n}(-1)^{n-k}\left(\begin{array}{l}
n \\
k
\end{array}\right) f(x+k h) .
$$


We then recall the definition of an $n$-additive function and its diagonalization along with their useful properties.

Definition 2 Let $n$ be a positive integer. A function $A_{n}: X \rightarrow Y$ will be called an $n$ additive function if it is additive in each of its arguments; i.e., for each $1 \leq i \leq n$ and for all $x_{1}, \ldots, x_{n}, y_{i} \in X$,

$$
A_{n}\left(x_{1}, \ldots, x_{i}+y_{i}, \ldots, x_{n}\right)=A_{n}\left(x_{1}, \ldots, x_{i}, \ldots, x_{n}\right)+A_{n}\left(x_{1}, \ldots, y_{i}, \ldots, x_{n}\right) .
$$

Lemma 3 Let $A_{n}: X^{n} \rightarrow Y$ be an n-additive function, where $n$ is a positive integer, and let $r$ be a rational number. Then, for all $x_{1}, \ldots, x_{n} \in X$,

$$
A_{n}\left(x_{1}, \ldots, r x_{i}, \ldots, x_{n}\right)=r A_{n}\left(x_{1}, \ldots, x_{i}, \ldots, x_{n}\right) .
$$

In particular, when $r=0, A_{n}\left(x_{1}, \ldots, 0, \ldots, x_{n}\right)=0$.

Definition 3 The diagonalization of an $n$-additive function $A_{n}: X^{n} \rightarrow Y$, where $n$ is a positive integer, is the function $A^{n}: X \rightarrow Y$ defined by

$$
A^{n}(x)=A_{n}(x, \ldots, x) \quad \text { for all } x \in X .
$$

Lemma 4 Let $A^{n}: X \rightarrow Y$ be the diagonalization of an n-additive function, where $n$ is a positive integer, and let $r$ be a rational number. Then, for all $x \in X$,

$$
A^{n}(r x)=r^{n} A^{n}(x)
$$

Definition 4 A function $f: X^{n} \rightarrow Y$ will be called symmetric if it is invariant under a permutation of its arguments; that is,

$$
f\left(x_{1}^{\prime}, \ldots, x_{n}^{\prime}\right)=f\left(x_{1}, \ldots, x_{n}\right)
$$

for all $x_{1}, \ldots, x_{n} \in X$ and $\left(x_{1}^{\prime}, \ldots, x_{n}^{\prime}\right)$ denotes any permutation of $\left(x_{1}, \ldots, x_{n}\right)$.

Lemma 5 Let $A^{n}: X \rightarrow Y$ be the diagonalization of a symmetric n-additive function, where $n$ is a positive integer. Then, for all $x, y \in X$,

$$
A^{n}(x+y)=\sum_{k=0}^{n}\left(\begin{array}{l}
n \\
k
\end{array}\right) A_{n}(\underbrace{x, \ldots, x}_{k}, \underbrace{y, \ldots, y}_{n-k}) .
$$

Later on, we can state the relation between an $n$-additive function and the difference operator of its diagonalization as shown below.

Theorem 5 Let $A_{n}: X^{n} \rightarrow Y$ be a symmetric n-additive function, where $n$ is a positive integer, and let $A^{n}: X \rightarrow Y$ be the diagonalization of $A_{n}$. If $m \geq n$ is an integer, then, for all $x, h_{1}, \ldots, h_{m} \in X$,

$$
\Delta_{h_{1} \cdots h_{m}} A^{n}(x)= \begin{cases}n ! A_{n}\left(h_{1}, \ldots, h_{n}\right) & \text { if } m=n, \\ 0 & \text { if } m>n .\end{cases}
$$


We thereafter define a polynomial function of order $n$ and then provide the consequent result often used.

Definition 6 Let $n$ be a nonnegative integer. A function $f: X \rightarrow Y$ which satisfies

$$
\Delta_{h}^{n+1} f(x)=0
$$

for all $x, h \in X$, will be called a polynomial function of order $n$.

Theorem 7 If $: X \rightarrow Y$ is a polynomial function of order $n$, then, for all $x, h_{1}, \ldots, h_{n+1} \in X$,

$$
\Delta_{h_{1}, \ldots, h_{n+1}} f(x)=0 .
$$

\section{Generalized polynomial functional equations}

In this section, we will show that the general solution of the proposed functional equation (1) is the diagonalization of a symmetric $n$-additive function.

Theorem 8 Let $X$ and $Y$ be two linear spaces. Let $n$ be a positive integer. A function $f$ : $X \rightarrow Y$ satisfies the functional equation (1) if and only if $f(x)=A^{n}(x)$ for all $x \in X$ where $A^{n}: X \rightarrow Y$ is the diagonalization of a symmetric n-additive function.

Proof Suppose a function $f: X \rightarrow Y$ satisfies (1). Define a function $A_{n}: X^{n} \rightarrow Y$ by

$$
A_{n}\left(x_{1}, \ldots, x_{n}\right)=\frac{1}{n !} \Delta_{x_{1} \cdots x_{n}} f(0) \quad \text { for all } x_{1}, \ldots, x_{n} \in X .
$$

Since the difference operators commute, it immediately follows that $A_{n}$ is symmetric under any permutation of its arguments. Next, we will show that $A_{n}$ is additive in the first argument. From the definition of $A_{n}$ and by the definition and the commutative property of the difference operators, we have

$$
\begin{aligned}
& A_{n}\left(x+y, x_{2}, \ldots, x_{n}\right)-A_{n}\left(x, x_{2}, \ldots, x_{n}\right)-A_{n}\left(y, x_{2}, \ldots, x_{n}\right) \\
& \quad=\frac{1}{n !}\left(\Delta_{x+y} \Delta_{x_{2} \cdots x_{n}} f(0)-\Delta_{x} \Delta_{x_{2} \cdots x_{n}} f(0)-\Delta_{y} \Delta_{x_{2} \cdots x_{n}} f(0)\right) \\
& \quad=\frac{1}{n !} \Delta_{x_{2} \cdots x_{n}}\left(\Delta_{x+y} f(0)-\Delta_{x} f(0)-\Delta_{y} f(0)\right) \\
& \quad=\frac{1}{n !} \Delta_{x_{2} \cdots x_{n}}(f(x+y)-f(x)-f(y)+f(0)) \\
& \quad=\frac{1}{n !} \Delta_{x_{2} \cdots x_{n}} \Delta_{x} \Delta_{y} f(0) .
\end{aligned}
$$

Noting that $\Delta_{h}^{n+1} f(x)=\Delta_{h}^{n} f(x+h)-\Delta_{h}^{n} f(x)=n ! f(h)-n ! f(h)=0$ for all $x, h \in X$, and, by virtue of Theorem 7, we have $\Delta_{x_{2} \cdots x_{n}} \Delta_{x} \Delta_{y} f(0)=0$, which in turn implies that

$$
A_{n}\left(x+y, x_{2}, \ldots, x_{n}\right)=A_{n}\left(x, x_{2}, \ldots, x_{n}\right)+A_{n}\left(y, x_{2}, \ldots, x_{n}\right)
$$

for all $x, y, x_{2}, \ldots, x_{n} \in X$. Thus, $A_{n}$ is additive in the first argument. Taking into account the symmetry of $A_{n}$, we conclude that $A_{n}$ is $n$-additive. 
Setting $x=0$ in (1) gives

$$
\Delta_{y}^{n} f(0)=n ! f(y)
$$

If we let $A^{n}: X \rightarrow Y$ be the diagonalization of $A_{n}$, that is, $A^{n}(x)=\frac{1}{n !} \Delta_{x}^{n} f(0)$ for all $x \in X$, then the above equation simply states that $f(x)=A^{n}(x)$ for all $x \in X$.

Conversely, if $f(x)=A^{n}(x)$ where $A^{n}: X \rightarrow Y$ is the diagonalization of a symmetric $n$ additive function, then Theorem 5 tells us that

$$
\Delta_{y}^{n} A^{n}(x)=n ! A^{n}(y)
$$

which yields the function equation (1). Thus, the proof is complete.

Theorem 8 proves that the general solution of the functional equation (1) is the diagonalization of a symmetric $n$-additive function. Hence, if a function $f: X \rightarrow Y$ satisfies (1), then, with the aid of Lemma 4, we have

$$
f(r x)=r^{n} f(x)
$$

for all rational numbers $r$ and for all $x \in X$.

\section{Generalized stability}

In this section, we aim to prove the stability of the functional equation (1). Let us start with some lemmas that will be used in the proof of the main theorem. It should be noted that we will adopt the usual extension of the binomial coefficients, that is $\left(\begin{array}{c}n \\ m\end{array}\right)=0$ for all integers $m>n \geq 0$.

Lemma 6 Let $m$ and $n$ be nonnegative integers. Then

$$
\sum_{i=0}^{m}(-1)^{i}\left(\begin{array}{c}
n \\
i
\end{array}\right)\left(\begin{array}{c}
n \\
m-i
\end{array}\right)= \begin{cases}0 & \text { if } m \text { is odd } \\
(-1)^{m / 2}\left(\begin{array}{c}
n \\
m / 2
\end{array}\right) & \text { if } m \text { is even }\end{cases}
$$

Proof Simply equate the coefficients of $x^{m}$ in both sides of the identity

$$
(1-x)^{n}(1+x)^{n}=\left(1-x^{2}\right)^{n}
$$

Lemma 7 Let $X$ and $Y$ be two linear spaces and let $f: X \rightarrow Y$ be a function. Then, for all $x \in X$,

$$
\Delta_{2 x}^{n} f(0)=\sum_{k=0}^{n}\left(\begin{array}{l}
n \\
k
\end{array}\right) \Delta_{x}^{n} f(k x) .
$$

Proof From Lemma 2, we have

$$
\sum_{k=0}^{n}\left(\begin{array}{l}
n \\
k
\end{array}\right) \Delta_{x}^{n} f(k x)=\sum_{k=0}^{n}\left(\begin{array}{l}
n \\
k
\end{array}\right) \sum_{l=0}^{n}(-1)^{n-l}\left(\begin{array}{l}
n \\
l
\end{array}\right) f(k x+l x) .
$$


Evaluate the sums over $k$ and $l$ by summing over $k+l$ and $l$. Thus,

$$
\sum_{k=0}^{n}\left(\begin{array}{l}
n \\
k
\end{array}\right) \Delta_{x}^{n} f(k x)=\sum_{m=0}^{2 n} \sum_{l=0}^{m}(-1)^{n-l}\left(\begin{array}{c}
n \\
m-l
\end{array}\right)\left(\begin{array}{l}
n \\
l
\end{array}\right) f(m x)
$$

By Lemma 6 , the inner sum over $l$ vanishes for all odd values of $m$, and the remaining sums for all even values of $m$ give

$$
\sum_{k=0}^{n}\left(\begin{array}{l}
n \\
k
\end{array}\right) \Delta_{x}^{n} f(k x)=\sum_{p=0}^{n}(-1)^{n-p}\left(\begin{array}{l}
n \\
p
\end{array}\right) f(2 p x)=\Delta_{2 x}^{n} f(0)
$$

Lemma 8 Let $\phi: X^{2} \rightarrow[0, \infty)$ be a function. If a function $f: X \rightarrow Y$ satisfies the inequality

$$
\left\|\Delta_{y}^{n} f(x)-n ! f(y)\right\| \leq \phi(x, y) \quad \text { for all } x, y \in X
$$

then

$$
\left\|\Delta_{2 x}^{n} f(0)-2^{n} \Delta_{x}^{n} f(0)\right\| \leq \sum_{k=1}^{n}\left(\begin{array}{l}
n \\
k
\end{array}\right) \phi(k x, x)+\left(2^{n}-1\right) \phi(0, x) \quad \text { for all } x \in X
$$

Proof From Lemma 7, we obtain, for every $x \in X$,

$$
\Delta_{2 x}^{n} f(0)-2^{n} \Delta_{x}^{n} f(0)=\sum_{k=0}^{n}\left(\begin{array}{l}
n \\
k
\end{array}\right) \Delta_{x}^{n} f(k x)-2^{n} \Delta_{x}^{n} f(0) .
$$

Using the identity $\sum_{k=0}^{n}\left(\begin{array}{l}n \\ k\end{array}\right)=2^{n}$, we will have

$$
\begin{aligned}
\Delta_{2 x}^{n} f(0)-2^{n} \Delta_{x}^{n} f(0) & =\sum_{k=0}^{n}\left(\begin{array}{l}
n \\
k
\end{array}\right)\left(\Delta_{x}^{n} f(k x)-n ! f(x)\right)-2^{n}\left(\Delta_{x}^{n} f(0)-n ! f(x)\right) \\
& =\sum_{k=1}^{n}\left(\begin{array}{l}
n \\
k
\end{array}\right)\left(\Delta_{x}^{n} f(k x)-n ! f(x)\right)-\left(2^{n}-1\right)\left(\Delta_{x}^{n} f(0)-n ! f(x)\right) .
\end{aligned}
$$

From the assumption in the lemma, $\left\|\Delta_{x}^{n} f(k x)-n ! f(x)\right\| \leq \phi(k x, x)$. Therefore,

$$
\left\|\Delta_{2 x}^{n} f(0)-2^{n} \Delta_{x}^{n} f(0)\right\| \leq \sum_{k=1}^{n}\left(\begin{array}{l}
n \\
k
\end{array}\right) \phi(k x, x)+\left(2^{n}-1\right) \phi(0, x) .
$$

We will now move on to the proof of the generalized stability of the functional equation (1).

Theorem 9 Let $X$ be a linear space and let $Y$ be a Banach space. Let $\phi: X^{2} \rightarrow[0, \infty)$ be a function and $n$ be a positive integer. Define a function $\tau: X \rightarrow[0, \infty)$ by

$$
\tau(x)=\frac{1}{2^{n} n !}\left(\phi(0,2 x)+\left(2^{n+1}-1\right) \phi(0, x)+\sum_{k=1}^{n}\left(\begin{array}{l}
n \\
k
\end{array}\right) \phi(k x, x)\right) \quad \text { for all } x \in X
$$


Moreover, assume that

$$
\sum_{i=0}^{\infty} \frac{\tau\left(2^{i} x\right)}{2^{i n}} \quad \text { converges and } \quad \lim _{m \rightarrow \infty} \frac{\phi\left(2^{m} x, 2^{m} y\right)}{2^{m n}}=0 \quad \text { for all } x, y \in X .
$$

If a function $f: X \rightarrow Y$ satisfies the inequality

$$
\left\|\Delta_{y}^{n} f(x)-n ! f(y)\right\| \leq \phi(x, y) \quad \text { for all } x, y \in X
$$

then there exists a unique function $A^{n}: X \rightarrow Y$ satisfying the functional equation (1) and

$$
\left\|f(x)-A^{n}(x)\right\| \leq \sum_{i=0}^{\infty} \frac{\tau\left(2^{i} x\right)}{2^{\text {in }}} \text { for all } x \in X
$$

In addition, $A^{n}(x)=\lim _{m \rightarrow \infty} \frac{f\left(2^{m} x\right)}{2^{m n}}$ for all $x \in X$.

Proof Assume all conditions in the theorem. For every $x \in X$, we have

$$
n !\left(f(2 x)-2^{n} f(x)\right)=\left(n ! f(2 x)-\Delta_{2 x}^{n} f(0)\right)+\left(\Delta_{2 x}^{n} f(0)-2^{n} \Delta_{x}^{n} f(0)\right)+2^{n}\left(\Delta_{x}^{n} f(0)-n ! f(x)\right) .
$$

By the inequality (7),

$$
\left\|\Delta_{2 x}^{n} f(0)-n ! f(2 x)\right\| \leq \phi(0,2 x) \quad \text { and } \quad\left\|\Delta_{x}^{n} f(0)-n ! f(x)\right\| \leq \phi(0, x) .
$$

By Lemma 8,

$$
\left\|\Delta_{2 x}^{n} f(0)-2^{n} \Delta_{x}^{n} f(0)\right\| \leq \sum_{k=1}^{n}\left(\begin{array}{l}
n \\
k
\end{array}\right) \phi(k x, x)+\left(2^{n}-1\right) \phi(0, x) .
$$

Therefore,

$$
\begin{aligned}
\left\|f(2 x)-2^{n} f(x)\right\| \leq & \frac{1}{n !}\left(\left\|\Delta_{2 x}^{n} f(0)-n ! f(2 x)\right\|+\left\|\Delta_{2 x}^{n} f(0)-2^{n} \Delta_{x}^{n} f(0)\right\|\right. \\
& \left.+2^{n}\left\|\Delta_{x}^{n} f(0)-n ! f(x)\right\|\right) \\
\leq & \frac{1}{n !}\left(\phi(0,2 x)+\sum_{k=1}^{n}\left(\begin{array}{l}
n \\
k
\end{array}\right) \phi(k x, x)+\left(2^{n}-1\right) \phi(0, x)+2^{n} \phi(0, x)\right) \\
= & 2^{n} \tau(x) .
\end{aligned}
$$

Dividing the above inequality by $2^{n}$, we arrive at

$$
\left\|\frac{f(2 x)}{2^{n}}-f(x)\right\| \leq \tau(x) \quad \text { for all } x \in X
$$

We can now follow the standard steps to prove the stability. Let $m \geq 1$ be an integer. For every $x \in X$,

$$
\left\|\frac{f\left(2^{m} x\right)}{2^{m n}}-f(x)\right\|=\left\|\sum_{i=0}^{m-1}\left(\frac{f\left(2^{i+1} x\right)}{2^{(i+1) n}}-\frac{f\left(2^{i} x\right)}{2^{i n}}\right)\right\| \leq \sum_{i=0}^{m-1} \frac{1}{2^{i n}}\left\|\frac{f\left(2^{i+1} x\right)}{2^{n}}-f\left(2^{i} x\right)\right\| .
$$


Using inequality (9), we get

$$
\left\|\frac{f\left(2^{m} x\right)}{2^{m n}}-f(x)\right\| \leq \sum_{i=0}^{m-1} \frac{\tau\left(2^{i} x\right)}{2^{i n}}
$$

From the conditions (6), we finally have $\left\|\frac{f\left(2^{m} x\right)}{2^{m n}}-f(x)\right\| \leq \sum_{i=0}^{\infty} \frac{\tau\left(2^{i} x\right)}{2^{i n}}$. Next, we will show that the sequence $\left\{\frac{f\left(2^{m} x\right)}{2^{m n}}\right\}_{m=0}^{\infty}$ is a Cauchy sequence. For any integers $k, l>0$, using inequality (9), we have

$$
\left\|\frac{f\left(2^{k+l} x\right)}{2^{(k+l) n}}-\frac{f\left(2^{k} x\right)}{2^{k n}}\right\|=\frac{1}{2^{k n}}\left\|\frac{f\left(2^{l} \cdot 2^{k} x\right)}{2^{l n}}-f\left(2^{k} x\right)\right\| \leq \sum_{i=0}^{\infty} \frac{\tau\left(2^{i+k} x\right)}{2^{(i+k) n}} .
$$

From the conditions (6), the rightmost term in the above inequality vanishes when $k \rightarrow \infty$, which implies that the sequence $\left\{\frac{f\left(2^{m} x\right)}{2^{m n}}\right\}_{m=0}^{\infty}$ is a Cauchy sequence in the Banach space $Y$. Hence, we can define a function $A^{n}: X \rightarrow Y$ by

$$
A^{n}(x)=\lim _{m \rightarrow \infty} \frac{f\left(2^{m} x\right)}{2^{m n}} \quad \text { for all } x \in X
$$

From the inequality (10), if we let $m \rightarrow \infty$, then we obtain the inequality (8).

Next, we will show that the function $A^{n}$ satisfies the functional equation (1). Replacing $x$ and $y$, respectively, by $2^{m} x$ and $2^{m} y$ in the inequality (7) and dividing the result by $2^{m n}$, we obtain

$$
\left\|\frac{1}{2^{m n}} \Delta_{2^{m} y}^{n} f\left(2^{m} x\right)-n ! \frac{f\left(2^{m} y\right)}{2^{m n}}\right\| \leq \frac{\phi\left(2^{m} x, 2^{m} y\right)}{2^{m n}} .
$$

Recalling the definition of $A^{n}$ and noting that

$$
\begin{aligned}
\lim _{m \rightarrow \infty} \frac{1}{2^{m n}} \Delta_{2^{m} y}^{n} f\left(2^{m} x\right) & =\lim _{m \rightarrow \infty} \frac{1}{2^{m n}} \sum_{k=0}^{n}(-1)^{n-k}\left(\begin{array}{l}
n \\
k
\end{array}\right) f\left(2^{m}(x+k y)\right) \\
& =\sum_{k=0}^{n}(-1)^{n-k}\left(\begin{array}{l}
n \\
k
\end{array}\right) A^{n}(x+k y)=\Delta_{y}^{n} A^{n}(x) .
\end{aligned}
$$

Now taking the limit of (11) as $m \rightarrow \infty$ and using the conditions (6), we will see that $A^{n}$ indeed satisfies the functional equation (1).

We still need to prove the uniqueness of $A^{n}$. Assume that there exists another function $\tilde{A}^{n}: X \rightarrow Y$ which satisfies the functional equation (1) as well as the inequality (8). We have already shown in Theorem 8 that a solution of the functional equation (1) satisfies (5), that is, for every rational number $r$ and for every $x \in X$,

$$
A^{n}(r x)=r^{n} A^{n}(x) \text { and } \tilde{A}^{n}(r x)=r^{n} \tilde{A}^{n}(x) .
$$

Hence, for every positive integer $m$,

$$
\begin{aligned}
\left\|A^{n}(x)-\tilde{A}^{n}(x)\right\| & =\frac{1}{2^{m n}}\left\|A^{n}\left(2^{m} x\right)-\tilde{A}^{n}\left(2^{m} x\right)\right\| \\
& \leq \frac{1}{2^{m n}}\left(\left\|A^{n}\left(2^{m} x\right)-f\left(2^{m} x\right)\right\|+\left\|f\left(2^{m} x\right)-\tilde{A}^{n}\left(2^{m} x\right)\right\|\right)
\end{aligned}
$$




$$
\begin{aligned}
& \leq \frac{2}{2^{m n}} \sum_{i=0}^{\infty} \frac{\tau\left(2^{i} \cdot 2^{m} x\right)}{2^{i n}} \\
& =2 \sum_{i=0}^{\infty} \frac{\tau\left(2^{i+m} x\right)}{2^{(i+m) n}}
\end{aligned}
$$

Taking the limit as $m \rightarrow \infty$, and using the conditions (6), we will have $A^{n}(x)=\tilde{A}^{n}(x)$ which asserts the uniqueness of $A^{n}$. This completes the proof.

We will also give a stability theorem with the conditions slightly different from those in Theorem 9.

Theorem 10 Let $X$ be a linear space and let $Y$ be a Banach space. Let $\phi: X^{2} \rightarrow[0, \infty)$ be a function and $n$ be a positive integer. Define a function $\tau: X \rightarrow[0, \infty)$ by

$$
\tau(x)=\frac{1}{2^{n} n !}\left(\phi(0,2 x)+\left(2^{n+1}-1\right) \phi(0, x)+\sum_{k=1}^{n}\left(\begin{array}{l}
n \\
k
\end{array}\right) \phi(k x, x)\right) \text { for all } x \in X
$$

Moreover, assume that

$$
\sum_{i=1}^{\infty} 2^{i n} \tau\left(2^{-i} x\right) \quad \text { converges and } \lim _{m \rightarrow \infty} 2^{m n} \phi\left(2^{-m} x, 2^{-m} y\right)=0 \quad \text { for all } x, y \in X
$$

If a function $f: X \rightarrow Y$ satisfies the inequality

$$
\left\|\Delta_{y}^{n} f(x)-n ! f(y)\right\| \leq \phi(x, y) \quad \text { for all } x, y \in X
$$

then there exists a unique function $A^{n}: X \rightarrow Y$ satisfying the functional equation (1) and

$$
\left\|f(x)-A^{n}(x)\right\| \leq \sum_{i=1}^{\infty} 2^{i n} \tau\left(2^{-i} x\right) \quad \text { for all } x \in X
$$

In addition, $A^{n}(x)=\lim _{m \rightarrow \infty} 2^{m n} f\left(2^{-m} x\right)$ for all $x \in X$.

Proof We can follow the beginning of the proof of Theorem 9. But the inequality (9) will be replaced by

$$
\left\|2^{n} f\left(\frac{x}{2}\right)-f(x)\right\| \leq 2^{n} \tau\left(\frac{x}{2}\right) \quad \text { for all } x \in X
$$

which will ends up at a bound similar to (10), that is,

$$
\left\|2^{m n} f\left(2^{-m} x\right)-f(x)\right\| \leq \sum_{i=1}^{\infty} 2^{i n} \tau\left(2^{-i} x\right)
$$

The rest of the proof can be carried out in the same fashion as that of Theorem 9 .

The following corollary states the Hyers-Ulam stability of the functional equation (1). 
Corollary 1 Let $X$ be a linear space and let $Y$ be a Banach space. Let $n$ be a positive integer and let $\varepsilon>0$ be a constant. If a function $f: X \rightarrow Y$ satisfies the inequality

$$
\left\|\Delta_{y}^{n} f(x)-n ! f(y)\right\| \leq \varepsilon \quad \text { for all } x, y \in X,
$$

then there exists a unique function $A^{n}: X \rightarrow Y$ satisfying the functional equation (1) and

$$
\left\|f(x)-A^{n}(x)\right\| \leq\left(\frac{3 \cdot 2^{n}-1}{\left(2^{n}-1\right) n !}\right) \varepsilon \quad \text { for all } x \in X .
$$

Proof The function $\phi(x, y)=\varepsilon$ satisfies the conditions in Theorem 9 and we will get $\tau(x)=\frac{3 \cdot 2^{n}-1}{2^{n} n !} \varepsilon$. Hence, there exists a unique function $A^{n}: X \rightarrow Y$ satisfying the functional equation (1) with the bound

$$
\left\|f(x)-A^{n}(x)\right\| \leq \sum_{i=0}^{\infty} \frac{\tau\left(2^{i} x\right)}{2^{i n}}=\left(\frac{3 \cdot 2^{n}-1}{\left(2^{n}-1\right) n !}\right) \varepsilon .
$$

Finally, we will give a result related to the problem extended by T. Aoki and Th. M. Rassias in the following corollary.

Corollary 2 Let $X$ be a linear space and let $Y$ be a Banach space. Let $n$ be a positive integer, let $\varepsilon>0$ be a constant and let $p>0$ be a constant with $p \neq n$. If a function $f: X \rightarrow Y$ satisfies the inequality

$$
\left\|\Delta_{y}^{n} f(x)-n ! f(y)\right\| \leq \varepsilon\left(\|x\|^{p}+\|y\|^{p}\right) \quad \text { for all } x, y \in X,
$$

then there exists a unique function $A^{n}: X \rightarrow Y$ satisfying the functional equation (1) and

$$
\left\|f(x)-A^{n}(x)\right\| \leq\left(\frac{2^{p}+3 \cdot 2^{n}-2+\sum_{k=1}^{n}\left(\begin{array}{l}
n \\
k
\end{array}\right) k^{p}}{\left(2^{n}-2^{p}\right) n !}\right) \varepsilon\|x\|^{p} \quad \text { for all } x \in X .
$$

Proof The function $\phi(x, y)=\varepsilon\left(\|x\|^{p}+\|y\|^{p}\right)$ satisfies the conditions (6) in Theorem 9 when $0<p<n$ and satisfies the conditions (12) in Theorem 10 when $p>n$. In either case, we will have

$$
\tau(x)=\left(\frac{2^{p}+3 \cdot 2^{n}-2+\sum_{k=1}^{n}\left(\begin{array}{l}
n \\
k
\end{array}\right) k^{p}}{2^{n} n !}\right) \varepsilon\|x\|^{p} .
$$

Once we evaluate the bound according to Theorem 9 and Theorem 10, we will get the result as desired in the corollary.

\section{Competing interests}

The authors declare that they have no competing interests.

\section{Authors' contributions}

PN initiated the study and conceived the way of proofs. TE carried out the results and drafted the manuscript. PU verified mathematical correctness of proofs. All authors read and approved the final manuscript.

\section{Acknowledgements}

We are very grateful to the referees for their valuable suggestions that improved this article. 


\section{References}

1. Ulam, SM: A Collection of Mathematical Problems. Interscience, New York (1968)

2. Hyers, DH: On the stability of the linear functional equation. Proc. Natl. Acad. Sci. USA 27, $222-224$ (1941)

3. Aoki, T: On the stability of the linear transformation in Banach spaces. J. Math. Soc. Jpn. 2, 64-66 (1950)

4. Rassias, TM: On the stability of linear mappings in Banach spaces. Proc. Am. Math. Soc. 72, 297-300 (1978)

5. Gavruta, P: A generalization of the Hyers-Ulam-Rassias stability of approximately additive mappings. J. Math. Anal. Appl. 184, 431-436 (1994)

6. Forti, GL: Hyers-Ulam stability of functional equations in several variables. Aequ. Math. 50, 143-190 (1995)

7. Park, W, Bae, J: On a Cauchy-Jensen functional equation and its stability. J. Math. Anal. Appl. 323, 634-643 (2006)

8. Lee, Y, Chung, S: Stability of a quadratic Jensen type functional equation in the spaces of generalized functions. J. Math. Anal. Appl. 324, 1395-1406 (2006)

9. Chang, I, Jung, Y: Stability for the functional equation of cubic type. J. Math. Anal. Appl. 334, $85-96$ (2007)

10. Najati, A: On the stability of a quartic functional equation. J. Math. Anal. Appl. 340, 569-574 (2008)

11. Gilányi, A: Hyers-Ulam stability of monomial functional equations on a general domain. Proc. Natl. Acad. Sci. USA 96 10588-10590 (1999)

12. Czerwik, S: Functional Equations and Inequalities in Several Variables. World Scientific, Singapore (2002)

doi:10.1186/1687-1847-2012-135

Cite this article as: Eungrasamee et al.: Generalized stability of classical polynomial functional equation of order $n$. Advances in Difference Equations 2012 2012:135.

\section{Submit your manuscript to a SpringerOpen ${ }^{\circ}$ journal and benefit from:}

- Convenient online submission

Rigorous peer review

- Immediate publication on acceptance

- Open access: articles freely available online

- High visibility within the field

- Retaining the copyright to your article 